\title{
Diagnóstico de las necesidades de capacitación de docentes de biología, química, física y matemática, en áreas disciplinares, pedagógicas, y uso de las tecnologías para la promoción de habilidades de pensamiento científico
}

\author{
Diagnosis of Training Needs of Teachers of Biology, Chemistry, Physics, and Mathematics, in \\ Disciplinary, Pedagogical Areas, and Use of Technologies to Promote Scientific Thinking Skills
}

Diagnóstico das necessidades de capacitação de docentes de biologia, química, física e matemática, em áreas disciplinares, pedagógicas, e uso das tecnologias para a promoção de habilidades de pensamento científico

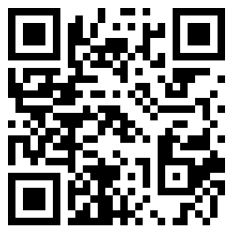

Adriana Zúñiga-Meléndez

Universidad Nacional

ROR:: ROR: https://ror.org/01t466c14 Facultad de las Ciencias Exactas y Naturales

Escuela de Ciencias Biológicas Heredia, Costa Rica adrianakamu@yahoo.es

D http://orcid.org/0000-0002-7911-2288

Alejandro Durán-Apuy

Universidad Nacional

ROR:: ROR: https://ror.org/01t466c14 Facultad de las Ciencias Exactas y Naturales

Escuela de Ciencias Biológicas Heredia, Costa Rica alejandro_d_a@yahoo.com D http://orcid.org/0000-0001-6605-6048

Jesennia Chavarría-Vásquez

Universidad Nacional

ROR:: ROR: https://ror.org/01t466c14 Facultad de las Ciencias Exactas y Naturales Escuela de Matemática Heredia, Costa Rica jcha@una.cr

http://orcid.org/0000-0002-6112-1231 
http://doi.org/10.15359/ree.24-3.23

http://www.una.ac.cr/educare

educare@una.ac.cr

\section{Ronny Gamboa-Araya} Universidad Nacional

ROR:: ROR: https://ror.org/01t466c14

Facultad de las Ciencias Exactas y Naturales

Escuela de Matemática

Heredia, Costa Rica

ronny.gamboa.araya@una.cr

iD http://orcid.org/0000-0002-9531-0372

\section{Ana Francis Carballo-Arce}

Universidad Nacional

ROR:: ROR: https://ror.org/01t466c14

Facultad de las Ciencias Exactas y Naturales

Escuela de Química

Heredia, Costa Rica

anafrancis01@hotmail.com

iD http://orcid.org/0000-0002-5379-8912

Xinia Vargas-González

Universidad Nacional

ROR:: ROR: https://ror.org/01t466c14

Facultad de las Ciencias Exactas y Naturales,

Escuela de Química

Heredia, Costa Rica

xiniavargas.gonzalez@gmail.com

D http://orcid.org/0000-0002-1097-2394

Nelson Campos-Quesada

Ministerio de Educación Pública

San José, Costa Rica

nelson.campos.quesada@mep.go.cr

(iD) http://orcid.org/0000-0002-2926-4523

Cecilia Sevilla-Solano

Ministerio de Educación Pública

San José, Costa Rica

cecilia.sevilla.solano@mep.go.cr

(iD http://orcid.org/0000-0002-3806-6772

Isabel Torres-Salas

Universidad Nacional

ROR:: ROR: https://ror.org/01t466c14 Centro de Investigación Docencia y Educación

División de Educología

Heredia, Costa Rica

isabeltorrescr96@gmail.com

http://orcid.org/0000-0003-2568-929X

Adriana Zúñiga-Meléndez, Alejandro Durán-Apuy, Jesennia Chavarría-Vásquez, Ronny Gamboa-Araya, Ana Francis Carballo-Arce, Xinia Vargas-González, Nelson Campos-Quesada, Cecilia Sevilla-Solano e Isabel Torres-Salas 
Recibido • Received • Recebido: 06 / 03 / 2019

Corregido • Revised • Revisado: 15 / 07 / 2020

Aceptado • Accepted • Aprovado: 18 / 08 / 2020

Resumen: Este estudio tiene como objetivo conocer las necesidades de capacitación del personal docente de ciencias naturales (biología, química y física) y de ciencias exactas (matemática), de colegios públicos académicos diurnos de la Dirección Regional de Educación de Heredia, para el desarrollo de habilidades científicas. Se trabajó con 72 docentes de matemática y 88 de ciencias naturales. La investigación se realizó desde el paradigma naturalista, con un enfoque cualitativo dominante y con un diseño del tipo de estudio fenomenológico. Se utilizó un instrumento tipo cuestionario, que tenía 12 preguntas dirigidas a indagar las necesidades de capacitación sobre los saberes pedagógicos y el uso de las tecnologías, y se hizo una exploración acerca del grado de interés que tiene el personal docente por recibir capacitación en saberes disciplinares. Las categorías utilizadas para el análisis son las siguientes: aspectos generales del personal docente en ejercicio; intereses de capacitación en el campo disciplinar, pedagógico y uso de las tecnologías; así como retos manifestados. Entre los principales hallazgos se evidenció el interés del cuerpo docente para participar en procesos de actualización en temas como la planificación, mediación y evaluación; además, en el uso de las tecnologías de la información y la comunicación. Además, se determinó que la capacitación en áreas disciplinarias debe estar enfocada al diseño de estrategias de mediación que permitan abarcar los temas de los programas de forma interdisciplinaria y contextualizada, así como en el diseño de procesos evaluativos que permitan evidenciar, en el estudiantado, el grado de apropiación de las habilidades de pensamiento científico y matemático.

Palabras claves: Enseñanza de las ciencias naturales; enseñanza de las matemáticas; actualización profesional; habilidades; educación científica; habilidades de pensamiento científico; pedagogía y tecnologías de la información.

Abstract: This study aims to better understand training secondary and high school teachers require to develop scientific thinking skills in the areas of natural science (Biology, Chemistry, and Physics) and Mathematics. The study focuses on teachers of these disciplinary areas from public schools with an academic curriculum of the Heredia District of Education in Costa Rica. The study worked with 72 teachers of Mathematics and 88 teachers of natural sciences. The research used a naturalistic paradigm. The experimental design used a dominant qualitative approach, combined with a phenomenological study type. The study developed a questionnaire-type instrument to gather the information needed. This instrument consisted of a total of 12 questions in the following areas: pedagogical knowledge and use of technologies, and exploration of the degree of interest that teaching staff has in receiving training in disciplinary knowledge. The five categories used to analyze the teachers' answers to the instrument were the following: general aspects of the practicing teacher, training interests in the disciplinary and pedagogical field, use of technologies, and challenges expressed by the teaching staff. Among the main findings of the study, the interest of the teaching staff to participate in updating processes in topics such as planning, mediation, and evaluation was evident, as well as training in the use of information and communication technologies. Also, it was determined that training in disciplinary areas should focus on the design of mediation strategies that allow the contents of the programs to be covered in an interdisciplinary and contextualized way. The teachers also showed interest in receiving training related to the design of evaluative processes that allow giving appropriate feedback to students, in terms of the degree of appropriation of scientific and mathematical thinking skills. 
http://doi.org/10.15359/ree.24-3.23

http://www.una.ac.cr/educare

educare@una.ac.cr

Keywords: Natural science teaching; mathematics teaching; professional update; skills; scientific education; scientific thinking skills; pedagogy and information technologies.

Resumo: O objetivo deste estudo foi conhecer as necessidades de treinamento que tem os docentes nos termos do desenvolvimento das habilidades científicas na área das ciências naturais (Biologia, Química e Física) e ciências exatas (Matemática), nas escolas diurnas da Direção Regional da província de Heredia segundo o sistema de edução pública da Costa Rica. O estudo foi desenvolvido no âmbito do paradigma naturalista, com abordagem qualitativo dominante e com delineamento do tipo de estudo fenomenológico. No desenvolvimento da pesquisa foi utilizado um instrumento tipo questionário, com 12 perguntas para validar o conhecimento atual e detetar necessidades de treinamento no uso das tecnologias, além de explorar o grau de interesse que têm os docentes para receber treinamento neste campo disciplinar. Para tal fim, foram consideradas as seguintes categorias de análise: aspetos gerais do docente, interesse do docente tanto no campo disciplinar como no contexto pedagógico e no uso das tecnologias; finalmente foram considerados os desafios inerentes que poderia representar este processo para eles. Na aplicação do questionário foram escolhidos 72 professores de matemática e 88 professores das ciências naturais. A partir dos resultadados obtidos encontrou-se uma evidente disposição do corpo docente para participar em processos de atualização dirigidos ao fortalecimento em temas de planejamento, mediação e avaliação das aulas no uso de tecnologias da informação e comunicação. Além disso, determinou-se que o treinamento em áreas disciplinares deveria focar-se no desenho de estratégias de mediação que permitiam abordar os tópicos dos programas de estudo num processo interdisciplinar e melhor contextualizado, bem como validar adequadamente os processos de avaliação que permitissem medir o grau de apropriação de habilidades de pensamento científico e matemático.

Palavras-chave: ensino de ciências naturais; ensino de matemática; atualização profissional; habilidades; educação científica; habilidades de pensamento científico; pedagogia e tecnologias da informação.

\section{Introducción}

El informe "La condición de la educación en matemáticas y ciencias naturales en América Latina y el Caribe (ALC)", elaborado por el Banco Interamericano de Desarrollo (BID) en el 2010 (Valverde y Näslund Hadley, 2010), describe de forma detallada la condición de la educación en matemática y ciencias naturales en esta región, exponiendo las deficiencias presentes en el proceso de enseñanza y aprendizaje de estas áreas disciplinares.

Las investigaciones sobre las oportunidades para aprender que están disponibles para los estudiantes en la región ALC presentan un panorama perturbador. Los jóvenes no están quedando preparados apropiadamente para cumplir los requisitos de matemáticas y ciencias naturales que exige una economía mundial que está cada vez más interconectada. Entre los causantes de esta situación se hallan los currículos débiles, materiales de aprendizaje inadecuados y la falta de dominio por parte de los docentes en matemáticas y en las ciencias naturales. (Valverde y Näslund Hadley, 2010, p 13) 
http://doi.org/10.15359/ree.24-3.23

Asimismo, señalan que, en el contexto de la enseñanza de las ciencias naturales y matemáticas, el cuerpo docente cuenta con una inadecuada formación en estas disciplinas, aunado a modelos pedagógicos centrados en la transmisión de conocimientos donde se fomenta poco el desarrollo del razonamiento científico y matemático.

A nivel de Costa Rica en el tercer informe del Estado de la Educación generado desde el Programa Estado de la Nación (2011), se puntualizaron diversos hallazgos sobre la educación científica, entre los que se señala: la enseñanza de las ciencias naturales en la educación diversificada ( $10^{\mathrm{mo}}$ y $11^{\mathrm{mo}}$ nivel escolar), al ser fragmentada, no permite la articulación de las temáticas, lo cual dificulta un abordaje metodológico coherente con la naturaleza de las disciplinas. Lo anterior se suma a un modelo pedagógico basado en un enfoque de contenidos fácticos, cuyo fin fundamental es la comprobación de conocimiento a través de las pruebas escritas o exámenes, tanto a nivel local como nacional, que deja de lado el desarrollo del pensamiento científico, en contradicción con lo que plantean los programas de estudio vigentes.

Así mismo, en el quinto informe del Estado de la Educación del Programa Estado de la Nación (2015), se señala el bajo rendimiento académico alcanzado en las pruebas estandarizadas, tales como PISA [el programa internacional para la Evaluación de Estudiantes o Informe PISA], donde se pone de manifiesto que en matemática cerca del $80 \%$ de la población estudiantil evaluada obtuvo una nota inferior a 70\%. Esta realidad se resalta de nuevo en el Sexto Informe Estado de la Educación correspondiente al (Programa Estado de la Nación, 2017) que destaca que: "el rendimiento en los exámenes PISA 2015 no fue inferior al de años anteriores. La mayoría de estudiantes obtiene bajas calificaciones, en especial en competencia matemática" (p. 50). Cabe resaltar que la pruebas PISA se transforman en un parámetro referenciador en tanto que tienen como objetivo evaluar las destrezas generales de jóvenes en habilidades de comprensión lectora, matemáticas y ciencias naturales.

Por su parte, el informe del Programa Estado de la Nación (2014) menciona que Costa Rica presenta una economía que comienza a implementar procesos de producción más eficientes y con calidad creciente, la cual impulsa el desarrollo de una ciudadanía participativa que requiere el fortalecimiento de la enseñanza de la matemática y las ciencias naturales, que promueva la comprensión de los procesos científicos y tecnológicos, la aplicación de diferentes saberes disciplinarios, así como los valores y habilidades para que la ciudadanía logre desenvolverse en la sociedad.

A nivel universitario se ha generado una preocupación creciente sobre el nivel académico con que ingresan el estudiantado en las diferentes universidades. Producto de esta situación, la Universidad Nacional ha realizado esfuerzos encaminados al diagnóstico de los conocimientos y habilidades científico-matemáticas que posee esta población estudiantil, mediante la ejecución de las pruebas de diagnóstico específicas realizadas en el área de matemática y química. A partir de los resultados obtenidos en el diagnóstico de matemática, únicamente el 2,5\% de estudiantes logró alcanzar una nota superior a $60 \%$, la mayoría de estudiantes (76,8\%) obtuvo calificaciones entre $20 \%$ y 40\% y el promedio de las calificaciones obtenidas fue de tan solo 29,73\% (Zamora Araya, 2015). 
http://doi.org/10.15359/ree.24-3.23

http://www.una.ac.cr/educare

educare@una.ac.cr

En el área de química, los resultados de este diagnóstico indican que del total de la muestra en el 2015, solamente un 6\%, obtuvo notas superiores a 70\%, de igual forma en el 2016 el porcentaje de estudiantes que superó la nota de $70 \%$ solamente fue de un $10 \%$. Estas deficiencias en la formación básica se ponen de manifiesto en los cursos iniciales afines a estas áreas, de las diferentes carreras, en los cuales la aprobación suele ser muy baja. Ante esta realidad educativa y como respuestas a las necesidades actuales expuestas desde el informe del Programa Estado de la Nación (2014), el Ministerio de Educación Pública (MEP), juntamente con el Consejo Superior de Educación, entes encargados de liderar las políticas educativas en Costa Rica, establecieron un cambio de programas de estudio para la educación secundaria. En el caso de matemática, a partir del 2016 se planteó un programa con un enfoque por competencias y, en el caso de ciencias naturales, se implementaron programas a partir del 2018 con un enfoque participativo.

[Estos programas] buscan acciones inter y transdisciplinarias para la formación integral del estudiantado al promover el desarrollo de habilidades para la comunicación, la capacidad crítica y reflexiva, mediante la aplicación de procesos propios de la ciencia, como plantear preguntas, explorar, experimentar, contrastar información y tomar decisiones para resolver problemas. (MEP, 2017a, p. 8)

Con la finalidad de lograr las acciones planteadas en la reforma curricular propuesta por el Ministerio de Educación Pública de Costa Rica, se recurre a estrategias propias del aprendizaje activo.

El aprendizaje activo es una aproximación metodológica centrada en el estudiantado, con la premisa de que el conocimiento se construye a partir de la interacción con los demás individuos, apoyándose en la reflexión y vivencias situadas en un contexto determinado, que busca desarrollar la capacidad de pensamiento crítico.

El personal docente es un agente facilitador y guía que busca la formación integral del individuo, estructurando actividades específicas de enseñanza que permitan una relación directa entre la práctica y la teoría en busca de la trascendencia y aplicación de los saberes en diferentes contextos y situaciones de su vida (Aristizabal-Almanza et al., 2018).

Lo anterior conlleva a un cambio de paradigma de una educación centrada en el contenido, a una centrada en el estudiantado. Desde este entendido resulta necesario determinar cuáles son las necesidades de formación pedagógicas y disciplinares que tiene el cuerpo docente que participa en la ejecución de los nuevos programas de estudio.

\section{Retos y desafíos del siglo XXI}

El siglo XXI está caracterizado por constantes cambios en los diversos campos que abarca un mundo globalizado, lo cual exige una transformación profunda y trascendental de la educación, donde los retos y desafíos requieren acceder a la información de manera rápida y eficaz (Bozu e Imbernón, 2016).

6 
http://doi.org/10.15359/ree.24-3.23

Según Palomares Ruiz (2004), este siglo estará marcado por la globalización como concepto emergente, por la imposición de modelos de vida y pensamiento transmitidos por diferentes medios de comunicación masiva, también será evidente el incremento en el avance tecnológico, además se dará un aumento del individualismo, la obsesión por la inmediatez y el paso de una sociedad tecnológica a una sociedad del conocimiento. Debido a este cambio hacia la sociedad del conocimiento, se prevé, según informe de seguimiento de la educación UNESCO (2017), que para el "2020, el mundo podría tener 40 millones menos de trabajadores con educación superior con respecto a la demanda" (p. xix). Ello implica grandes retos para las universidades.

De acuerdo con Klimenko (2008) el siglo XX se caracteriza por haber acelerado el mundo:

Trajo ... una aceleración de los avances en todos los niveles, desde la tecnología, la industria, la organización empresarial, los cuales a su vez revelaron la gravedad de problemáticas que amenazan la humanidad, empezando por la escasez de recursos energéticos o la inminente amenaza del calentamiento global, y terminando con problemáticas sociales, como pobreza, deterioro progresivo de valores, superficialidad de la filosofía consumista, etc. (p.192)

El siglo XXI ha heredado una serie de problemáticas donde la educación tiene un papel importante en la "búsqueda de un modelo holístico, endógeno y sostenible. Sin embargo, las tentativas de llevar a la realidad estas iniciativas, con sensibilidad ambiental y rostro humano, no han dado los resultados esperados" (Torres Salas, 2010, p.132).

Prueba de ello es que los actuales contextos de este mundo globalizado presentan grandes diferencias, que evidencian que las políticas educativas que pretenden la inclusión, la equidad, la erradicación del hambre, la construcción de una paz duradera, el desarrollo de competencias científicas para tener ciudadanías que resuelvan los problemas con un pensamiento crítico y reflexivo, no está llegando a las diferentes personas con la urgencia que se necesita, porque se evidencian "colosales problemáticas de pobreza, condiciones infrahumanas de vida, índices de muerte infantil por desnutrición, etc., que azotan a muchos países, aun hoy, en pleno siglo XXI, con todo el progreso tecnológico, científico y económico" (Klimenko, 2008, p. 193).

Según Bozu e Imbernón (2016), lo anterior hay que contextualizarlo en el momento actual histórico de la humanidad, en el que se requiere de cambios fundamentales en el pensar, actuar y ser de las personas, proceso en el cual la educación debe abocarse hacia la búsqueda de nuevos modelos pedagógicos, que den respuesta a los diferentes desafíos.

\section{Papel de la educación para responder a los desafíos del siglo XXI}

Como se ha venido mencionando, las consecuencias del desarrollo actual plantean desafíos y retos para el sistema educativo enmarcando en entornos cada vez más cambiantes y competitivos, en los que se genera la necesidad de estructuras y procesos sociales y organizacionales más flexibles, con alto desarrollo científico y tecnológico. 
http://doi.org/10.15359/ree.24-3.23

http://www.una.ac.cr/educare

educare@una.ac.cr

Ante estas situaciones, compete a la educación ofrecer alternativas de intervención y solución que permitan confrontary afrontar dichos desafíos y, para esto, se requiere implementar estrategias que faculten a las personas, las organizaciones y los grupos sociales para atender los retos que supone la sociedad moderna.

En este sentido, la educación debe garantizar una formación integral en las personas con la que se desarrollen competencias para la vida, se impulse la promoción de habilidades para el pensamiento crítico y sistémico, en consonancia con lo propuesto socialmente.

Así mismo, se requiere que la educación y los procesos de articulación de conocimiento generen las habilidades para la integración y uso de las tecnologías de la información que le permitan a las personas transitar en el mundo vertiginosamente cambiante e itinerante. En este sentido, se ha de garantizar la incorporación de las nuevas tecnologías de la información como contenidos curriculares y didácticos, a partir de las cuales se desarrollen habilidades para recabar información, contrastarla, retroalimentar procesos, hacer simulaciones y mantener la conectividad con la comunidad inmediata nacional e internacional.

Otro reto, no menos importante, es que el personal docente, para responder a las actuales transformaciones educativas, debe actualizarse, formarse y capacitarse tanto en aspectos disciplinares como pedagógicos, ya que los cambios permanentes, a su vez, originan el ejercicio de roles diferentes a los desempeñados en las décadas anteriores. Asimismo, las formas de la organización y estructura de los currículos se han flexibilizado de tal manera que habrá que adaptarse ellas, con elementos acordes para ofrecer mejores niveles de calidad en la formación educativa (Vélez Chablé, 2006). Esto nos lleva a reflexionar sobre las necesidades de capacitación o superación que tienen los grupos docentes como uno de los elementos clave para responder a los desafíos y demandas actuales de la educación.

\section{La educación científica}

En este contexto, la educación científica se transforma en un medio que promueve la formación de una ciudadanía planetaria, en tanto que facilita el desarrollo de habilidades de pensamiento para enfrentar las situaciones emergentes de una sociedad compleja e interrelacionada. En este sentido, Macedo (2016) apunta que la formación científica tiene un impacto en el desarrollo de las personas y la sociedad, dado que una población científicamente preparada posibilita que los individuos tomen decisiones y ejecuten acciones para un desarrollo sostenible, justo y equitativo.

Por tal razón, las instituciones educativas deben afrontar el desafío de brindar a cada persona la formación científica que le permita desempeñarse en el contexto actual, otorgándole capacidades para discernir, seleccionar e interpretar la información (Macedo, 2016; Peñaherrera León et al., 2013). De igual forma se le ha de otorgar una sólida formación en ciencias naturales 
http://doi.org/10.15359/ree.24-3.23

y exactas (matemática), para comprender y solucionar problemas complejos relacionados con la biología, física, química, ingeniería, medio ambiente, entre otros, a través del análisis de datos y el planteamiento de modelos matemáticos para su solución (Bosch et al., 2011).

En América Latina la educación científica se ha caracterizado por un aprendizaje cuya calidad educativa resulta insuficiente y donde las metodologías de enseñanza no han logrado despertar en el estudiantado el interés por las ciencias, lo que ha dificultado la promoción de vocaciones científicas y el interés por cursar carreras en áreas relacionadas con estas, y ha provocado la consecuente disminución de graduaciones universitarias en ellas (Bosch et al., 2011; Macedo, 2016).

Al respecto, Macedo (2016), Valverde y Näslund-Hadley (2010) señalan que los resultados de las pruebas PISA y otras evaluaciones internacionales, para los países de América Latina, ponen de manifiesto que la formación científica no ha sido significativa ni homogénea y que el desempeño del estudiantado se ubica por debajo del nivel óptimo. Apuntan algunos problemas que la afectan, tales como: una educación científica descontextualizada, basada en programas débiles, sin evidenciar relación entre los conocimientos y su aplicación en la vida cotidiana.

A nivel nacional, Alfaro Varela y Villegas (2010) señalan que la educación científica se ha desarrollado en forma fragmentada y poco articulada con otras asignaturas escolares, con una metodología que no favorece el desarrollo del pensamiento científico, sino que se centra en la preparación del estudiantado para aprobar pruebas escritas. En este sentido, Alfaro Varela y Villegas (2010), Valverde y Näslund-Hadley (2010) y Macedo (2016) apuntan que debe plantearse una reforma de la educación científica con base en tres preguntas fundamentales: qué se debe enseñar, a quiénes y cómo se debe enseñar, y que tome en cuenta aspectos tales como: una revisión de los currículos escolares, según las competencias que se desean desarrollar en el estudiantado; la promoción de procesos de aprendizaje que incentiven el pensamiento divergente, el aprender del error, el investigar, el argumentar, el aprender de otros seres y con estos; desarrollar la creatividad; promover el pensamiento y la comprensión científica

Estas demandas para la formación científica del estudiantado han promovido, en nuestro país, cambios en la estructura curricular, donde se entiende el currículo como el conjunto de elementos que se proponen para orientar lo que se enseña y cómo se enseña, y que contempla las distintas dimensiones del hecho educativo como lo son: la social, el contexto y lo pedagógico. Estos cambios se ven reflejados en los planes de estudio de la educación secundaria en las áreas de las ciencias naturales, así como de la matemática. No obstante, esta reformulación curricular debe ir acompañada de un cambio en la formación de docentes, en cuyos procesos de formación permanente se deben incluir nuevas metodologías de aprendizaje, a partir de la contextualización e indagación, el uso de recursos tecnológicos, y generar ambientes participativos para compartir experiencias y reflexionar acerca de sus propias prácticas. 
http://doi.org/10.15359/ree.24-3.23

http://www.una.ac.cr/educare

educare@una.ac.cr

\section{El currículo en el marco de la política educativa nacional de Ciencias Naturales y Matemática}

La política educativa La persona: Centro del proceso educativo y sujeto transformador de la sociedad (MEP, 2017b, p. 5) y la política curricular Educar para una nueva ciudadanía (MEP, 2015, p. 5) orientan las diferentes actividades y programas de estudio del sistema educativo costarricense, para promover el desarrollo de habilidades, entendidas como las capacidades para solucionar problemas y realizar tareas diversas, dentro de una pluralidad de condiciones, ambientes y situaciones. Estas habilidades se visualizan en los rasgos de los perfiles específicos del estudiantado en cada ciclo escolar y se distribuyen en dimensiones denominadas: maneras de pensar, formas de vivir en el mundo, formas de relacionarse con otros seres y herramientas para integrarse al mundo, para enriquecer el pensamiento crítico, el pensamiento sistémico, estilos de vida saludables, colaboración y comunicación, manejo de la información y tecnologías digitales, entre otras.

Desde esta perspectiva, el enfoque curricular de los programas de estudio aborda los pilares filosóficos del humanismo, racionalismo, constructivismo, socioconstructivismo, pedagogía crítica y habilidades para una nueva ciudadanía, para visualizar "el aprendizaje como una actividad social, en la cual, el estudiantado aprende a aprender en colaboración con los demás, intercambiando opiniones para la toma de decisiones que apoyan la solución de problemas" (MEP, 2017c, p. 15), de la comunidad, en el ámbito local y global.

Por su parte, el diseño curricular de los Programas de estudio de Ciencias que comprende física, química y biología desde sétimo hasta undécimo nivel, presenta ejes temáticos que organizan la articulación de los saberes propios de cada disciplina. Estos ejes se operacionalizan en criterios de evaluación, que establecen acciones ligadas a los aspectos de la cultura cotidiana y sistematizada, para prevenir, enfrentar y resolver situaciones de la vida diaria. Los criterios de evaluación son acompañados por situaciones de aprendizaje, que consisten en secuencias de diversas actividades, mediante las cuales se evalúa el progreso continuo del estudiantado en la construcción y apropiación de conocimientos y el desarrollo de habilidades para una nueva ciudadanía.

Estos programas fomentan diferentes habilidades "para la expresión y comprensión de ideas previas de su realidad, en contrastación con las ideas de otras personas y las vigentes en el ámbito científico" (MEP, 2017c, p. 17) y utilizan la metodología basada por la indagación. Respecto a la evaluación plantea:

La evaluación permite ... documentar y valorar las características y avance del estudiantado en el desarrollo de habilidades para una nueva ciudadanía. Cuando el estudiantado, socializa sus ideas previas, representa una evaluación diagnóstica sobre sus conocimientos y habilidades iniciales. Al continuar con la secuencia de situaciones de aprendizaje, se hace énfasis en el trabajo colaborativo, resolución de problemas presentes en la comunidad, se discuten y contrastan los hallazgos, y vuelven a revisar sus primeras ideas, lo que le permite conocer cómo ha evolucionado en su aprendizaje, considerando los aspectos atinentes a la evaluación formativa y sumativa. (MEP, 2017c, p. 24) 
http://doi.org/10.15359/ree.24-3.23

Por su parte, en cuanto a las ciencias exactas, los Programas de estudio de Matemática para la educación primaria y secundaria, vigentes a partir del 2012, tienen un diseño curricular por ejes disciplinares, cinco en total:

- La resolución de problemas como estrategia metodológica principal.

- La contextualización activa como un componente pedagógico especial.

- El uso inteligente y visionario de tecnologías digitales.

- La potenciación de actitudes y creencias positivas entorno a las matemáticas.

- El uso de la Historia de las Matemáticas. (MEP, 2012, p. 11)

Sin embargo, se enfatiza en la resolución de problemas en contextos reales como su enfoque central.

El nuevo diseño curricular se diferencia de los diseños anteriores basados en contenidos, en que reinterpreta el papel de los contenidos como organizadores del currículo y de las habilidades que alrededor de estos van a ser desarrolladas por el estudiantado. En este sentido se plantean cinco áreas matemáticas: números, medidas, geometría, relaciones y álgebra, estadística y probabilidad. Este diseño curricular se enfoca en el desarrollo de habilidades y competencias matemáticas, entendiendo la competencia matemática en los siguientes términos:

[La] capacidad del individuo para formular, emplear e interpretar las Matemáticas en una variedad de contextos. Incluye razonar matemáticamente y usar conceptos, procedimientos, hechos y herramientas para describir, explicar y predecir fenómenos. Ayuda a los individuos a reconocer el papel de las Matemáticas en el mundo y hacer juicios bien fundamentados y decisiones necesarias para ciudadanos constructivos, comprometidos y reflexivos. (OECD, 2010, p. 4 citado por MEP, 2012, p. 23)

Estas competencias matemáticas se apoyan para su desarrollo en procesos matemáticos, los cuales, según MEP (2012), son "actividades cognitivas (o tipos de actividades) que realizan las personas en las distintas áreas matemáticas y que se asocian a capacidades para la comprensión y usos de conocimientos" (p. 24). Esto es, el programa de estudio considera como centrales los procesos matemáticos de razonar y argumentar; plantear y resolver problemas; comunicar, conectar y representar.

El enfoque curricular que se asume es la resolución de problemas en contextos reales por medio de una mediación pedagógica con principios fundamentalmente constructivistas, que promueve la construcción del conocimiento por parte del estudiantado y el desarrollo de capacidades y habilidades en las áreas de matemáticas, tanto a nivel básico como de alta exigencia cognitiva. El plan de estudio establece, además, orientaciones metodológicas basadas en este enfoque por resolución de problemas, que sirven de guía o lineamientos para 
http://doi.org/10.15359/ree.24-3.23

http://www.una.ac.cr/educare

educare@una.ac.cr

la labor docente; además, la evaluación se concibe como una parte del proceso de enseñanza y aprendizaje, cuyo fin es obtener información válida y confiable que permita determinar el logro de habilidades, destrezas o competencias que se proponen en el plan de estudios.

Frente a este nuevo modelo curricular de las ciencias naturales y la matemática, es necesario clarificar aquellas necesidades del personal docente que le permitan atender oportunamente los cambios propuestos. Esto, partiendo del hecho que los cuerpos docentes fueron formados desde un modelo de educación centrado más en la enseñanza aprendizaje del contenido y que se enfrentan a una enseñanza centrada en el estudiantado y por habilidades.

Este nuevo modelo, que tiene como eje central el desarrollo de habilidades, supone para el cuerpo docente una restructuración de los procesos de planificación, mediación y evaluación de las experiencias de clase. Para esto resulta necesario realizar estrategia de acompañamiento que le permitan, al personal docente, fortalecer y apoyar esas prácticas pedagógicas y, a su vez, potenciar el desarrollo de habilidades para el pensamiento científico

\section{Metodología}

El objetivo principal de la investigación fue conocer las necesidades de capacitación del profesorado de ciencias exactas (matemática) y ciencias naturales (biología, física y química) de la educación secundaria. Esta se divide en tercer ciclo de educación general básica (EGB), conformada por los grados sétimo, octavo y noveno; y educación diversificada (ED) que contempla decimo y undécimo nivel. La investigación se llevó a cabo en los colegios públicos académicos diurnos en la Dirección Regional de Educación de Heredia, circuitos del 01 al 07, para el fortalecimiento del perfil docente en estos campos del conocimiento. En el 2018, la población de docentes en dichos circuitos de la Dirección Regional de Educación de Heredia estaba conformada por 98 docentes de matemática y 103 de ciencias naturales.

Lainvestigación se realizó con el paradigma naturalista, con un enfoquecualitativo dominante y con un diseño del tipo de estudio fenomenológico. Para recoger los datos se convocó al cuerpo docente, a través de autoridades educativas del Ministerio de Educación Pública, a una actividad académica, donde se aplicó en línea un instrumento, por área disciplinar, tipo cuestionario, con preguntas cerradas y abiertas. Además, se brindó un espacio para que los sujetos participantes manifestaran los retos y desafíos que enfrentan en el ejercicio de su práctica.

Del total de la población, asistieron a la convocatoria y estuvieron dispuestos a participar en la investigación 72 docentes de matemática y 88 de ciencias naturales, para un total de 160 docentes que imparten lecciones en el III ciclo de la Educación General Básica (EGB), así como en décimo y undécimo de la Educación Diversificada (ED).

De esta manera, la muestra participante estuvo conformada por un 55\% de profesorado que imparte asignaturas de ciencias naturales, química, física y biología y un $45 \%$ de profesorado 
http://doi.org/10.15359/ree.24-3.23

que imparte la asignatura de matemática; donde más del $60 \%$ del personal docente de ambas disciplinas poseían una experiencia superior a los 15 años en el campo de la docencia.

En cuanto al grado académico de las personas participantes, el 62,5\% de docentes de ciencias y un 66,7\% de matemática contaron con el grado académico de licenciatura. Se destaca, además, que el $23,9 \%$ en ciencias naturales y el $25 \%$ en matemática contaron con un posgrado. Esto indica que el personal docente se encuentra ubicado, según el escalafón profesional del Servicio Civil, en las dos categorías más altas (de seis) de la clasificación para profesorado de enseñanza media titulado (MT), alcanza un poco más del 30\% la escala MT5 y entre el 53\% y 59\% el MT6, tanto en ciencias naturales como en matemática, respectivamente.

El instrumento constaba de 12 preguntas dirigidas aindagar lasnecesidades decapacitación sobre los saberes pedagógicos. Tomaba en cuenta las siguientes dimensiones: planificación didáctica, mediación pedagógica y clima organizacional en el aula; búsqueda de información e investigación, y procedimientos de evaluación, así como hacer una exploración acerca del grado de interés que tiene el personal docente por recibir capacitación en saberes disciplinares. Con respecto a las habilidades relacionadas con la apropiación de las herramientas tecnológicas, se tomó en cuenta la utilización que hace el profesorado de los medios tecnológicos, así como las habilidades cognitivas con las que cuenta para el manejo de las TIC.

Para la elaboración de los instrumentos, un equipo interdisciplinario académico de la Facultad de Ciencias Exactas y Naturales (FCEN), del Centro en Investigación y Docencia en Educación (CIDE) de la Universidad Nacional de Costa Rica (UNA) y del Ministerio de Educación Pública (MEP), realizó un análisis de contenido de los programas deestudio vigentes de matemática y de ciencias naturales en el que se identificó el enfoque y diseño curricular, la metodología, la evaluación y la planificación que se propone en ellos, con el fin de conocer en qué medida tienen relación las necesidades de capacitación con las concepciones plasmadas en esos programas. El análisis y discusión de resultados se realizó alrededor de las siguientes categorías: aspectos generales del personal docente en ejercicio; intereses de capacitación en el campo disciplinar, pedagógico y uso de las tecnologías, así como retos manifestados por el cuerpo docente.

Los instrumentos fueron validados a través de un grupo focal; en este grupo participaron asesores y asesoras regionales de la gran área metropolitana, así como profesores y profesoras líderes de diferentes colegios y direcciones regionales. Los aspectos a validar fueron los siguientes: verificar que la información generada a partir de los instrumentos estuviera en concordancia con los objetivos de la investigación; si los ítems reunían las condiciones de claridad en términos de: instrucciones y el vocabulario utilizado; valorar la funcionalidad del orden y secuencia de las preguntas; determinar la duración real de la aplicación de la pruebas y, en el caso de las preguntas cerradas, evaluar si las categorías eran exhaustivas y mutuamente excluyentes.

Una vez analizadas las sugerencias y recomendaciones, las que se consideraron pertinentes se incluyeron en el instrumento final. 
http://doi.org/10.15359/ree.24-3.23

http://www.una.ac.cr/educare

educare@una.ac.cr

El análisis de resultados se basó en los intereses de capacitación del personal docente, en cuanto a: el saber disciplinar de acuerdo con cada disciplina (ciencias naturales o matemática), en el pedagógico y en el uso de las tecnologías; así como en los retos y desafíos de la práctica docente.

En cuanto a las preguntas cerradas, se presenta el porcentaje de respuesta de los ítems según las categorías establecidas para el grado de interés (alto, medio, bajo) o la frecuencia de respuesta. Para las preguntas abiertas, se hizo un análisis de contenido.

\section{Resultados}

El análisis de resultados se estructura considerando los siguientes apartados: aspectos generales del personal docente en ejercicio; intereses de capacitación en el saber disciplinar, en el saber pedagógico y en el uso de las tecnologías; así como los retos y desafíos de la práctica docente.

\section{Intereses de capacitación en el saber disciplinar, saber pedagógico y uso de las tecnologías}

\section{Saber disciplinar}

Los datos obtenidos del instrumento denominado "Saberes pedagógicos y tecnológicos", mostraron resultados muy variados, una parte del profesorado se limitó a dar respuestas muy generales que señalan, como temas de capacitación, áreas disciplinarias y no contenidos específicos; por lo anterior, se aplicaron filtros con la finalidad de valorar en cuales áreas de la química, física, biología y matemática, se requiere capacitación. De este proceso de selección, se determinaron un total de cinco tópicos para matemática, seis relacionados con biología, siete para física y nueve para química. Para cada temática se determinó el porcentaje respectivo, a partir de la cantidad de respuestas dadas.

Con respecto a matemática, se evidencia que la estadística y la probabilidad son los temas de mayor interés para capacitarse o recibir formación, registran un 33\% y un 26\%, respectivamente. Temas como geometría y funciones presentaron en conjunto un $29 \%$, y por último aparece algebra con un 12\% (Figura 1A).

En cuanto a biología, la mayoría de los tópicos estuvieron relacionados con la ecología (57\%), mientras que evolución, genética, célula y ciclo celular, así como el cuidado del medio ambiente, mostraron porcentajes entre el 9 y 10\%, en tanto que la biotecnología alcanzó el menor porcentaje con un 5\% (Figura 1B).

Por su parte, en física, el mayor interés de capacitación le corresponde al tema de cinemática con un 39,3\%, seguido por física moderna con 32,1\% y trabajo y energía con 14,3\%; electricidad, ondas y óptica también fueron temas de interés para las capacitaciones, pero no superaron el 7\% (Figura 1C). 
http://doi.org/10.15359/ree.24-3.23

http://www.una.ac.cr/educare educare@una.ac.cr

En el caso de química, se presentó una mayor cantidad de tópicos y una distribución más homogénea. Dentro de estos, destacan compuestos y enlaces químicos, ambos con un $19 \%$, seguidos por otros temas de interés como lo fueron estequiometría 15\% y química orgánica con $12 \%$, en los que se incluyen temas como nomenclatura de compuestos orgánicos y grupos funcionales (Figura 1D).

Figura 1: Temáticas de capacitación identificadas por los docentes de secundaria, A) Matemática,

B) Biología, C) Física y D) Química, Dirección Regional de Heredia, 2018

A

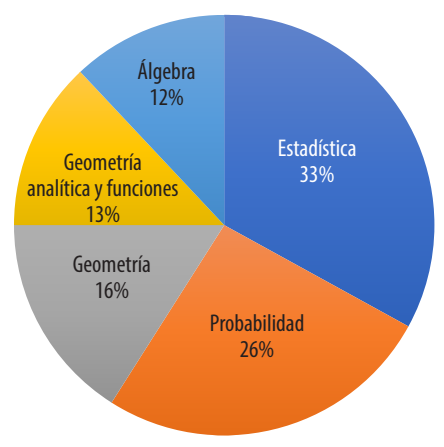

C

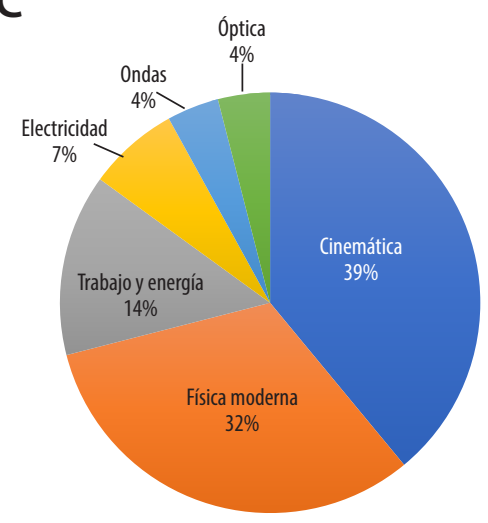

B

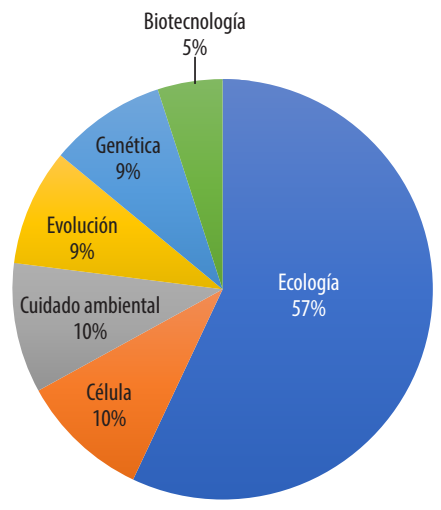

D

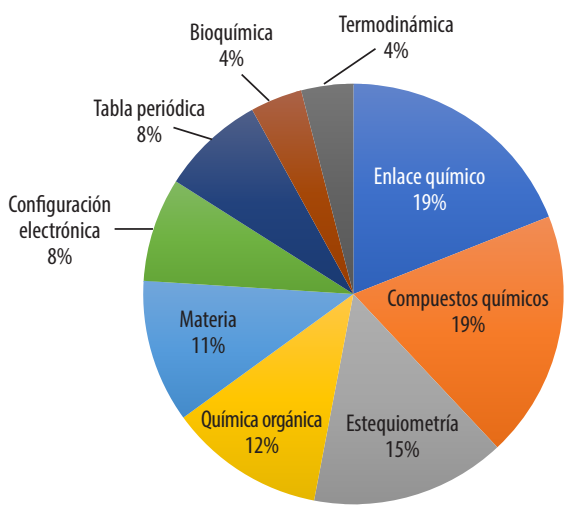

Nota: Elaboración propia. 
http://doi.org/10.15359/ree.24-3.23

http://www.una.ac.cr/educare

educare@una.ac.cr

\section{Saber pedagógico}

Los resultados obtenidos indican que los grupos docentes muestran interés por capacitarse en el campo pedagógico. En cuanto a la dimensión planificación didáctica, se puede afirmar que la tendencia en cuanto al grado de interés se ubica de medio a alto con un $90 \%$ o más en ambos aspectos contemplados, tanto en ciencias naturales como en matemática (Tabla 1).

Tabla 1: Grado de interés de docentes de ciencias naturales* y matemática en recibir capacitaciones en planificación didáctica, Dirección Regional de Educación de Heredia, 2018

\begin{tabular}{lccccccc}
\hline & \multicolumn{5}{c}{ Grado de interés } \\
\cline { 2 - 7 } \multicolumn{1}{c}{ Criterios } & \multicolumn{3}{c}{ Ciencias } & \multicolumn{3}{c}{ Matemática } \\
\cline { 2 - 7 } & $\begin{array}{c}\text { Alto } \\
\%\end{array}$ & $\begin{array}{c}\text { Medio } \\
\%\end{array}$ & $\begin{array}{c}\text { Bajo } \\
\%\end{array}$ & $\begin{array}{c}\text { Alto } \\
\%\end{array}$ & $\begin{array}{c}\text { Medio } \\
\%\end{array}$ & $\begin{array}{c}\text { Bajo } \\
\%\end{array}$ \\
\hline $\begin{array}{l}\text { Planificación didáctica contextualizada con } \\
\text { metodologías activas que le permitan al estudiantado } \\
\text { el desarrollo de habilidades para la argumentación, el }\end{array}$ & 58,0 & 33,0 & 9,0 & 68,0 & 27,8 & 4,2 \\
debate, así como la criticidad. & & & & & & & \\
$\begin{array}{l}\text { Planificación didáctica con coherencia entre las } \\
\text { situaciones de aprendizaje y los indicadores de } \\
\text { evaluación. Consideración de las características y } \\
\text { necesidades individuales del estudiantado. }\end{array}$ & 65,9 & 30,7 & 3,4 & 66,7 & 33,3 & 0,0 \\
\hline
\end{tabular}

*El término ciencias incluye a los docentes de ciencias, biología, física y química.

Nota: Cuestionario de docentes de ciencias naturales y matemática. Elaboración propia.

En esta misma línea, los resultados referentes a la dimensión mediación pedagógica, muestran que el interés del cuerpo docente por capacitarse tiene un comportamiento muy similar a la dimensión analizada con anterioridad (Tabla 1), ya que la tendencia en términos generales en todas las opciones es de medio a alto con un porcentaje mayor al $95 \%$ en todos los casos. De ahí que se puede decir que existe consenso en el personal docente en cuanto a recibir capacitación tanto en actividades de mediación pedagógica como en situaciones de aprendizaje que contribuyan a que el estudiantado desarrolle el pensamiento crítico y sistémico (Tabla 2). 
http://doi.org/10.15359/ree.24-3.23

http://www.una.ac.cr/educare educare@una.ac.cr

Tabla 2: Grado de interés de docentes de ciencias naturales * y matemática en recibir capacitaciones en mediación pedagógica, Dirección Regional de Educación de Heredia, 2018

\begin{tabular}{llccccc}
\hline & \multicolumn{5}{c}{ Grado de interés } \\
\cline { 2 - 7 } & \multicolumn{5}{c}{ Ciencias } & \multicolumn{3}{c}{ Matemática } \\
\cline { 2 - 7 } Criterios & $\begin{array}{c}\text { Alto } \\
\%\end{array}$ & $\begin{array}{c}\text { Medio } \\
\%\end{array}$ & $\begin{array}{c}\text { Bajo } \\
\%\end{array}$ & $\begin{array}{c}\text { Alto } \\
\%\end{array}$ & $\begin{array}{c}\text { Medio } \\
\%\end{array}$ & $\begin{array}{c}\text { Bajo } \\
\%\end{array}$ \\
\hline $\begin{array}{l}\text { Actividades de mediación pedagógica, utilizando la } \\
\text { metodología de indagación u otras metodologías } \\
\text { activas como la resolución de problemas, el aprendizaje }\end{array}$ & 61,4 & 36,4 & 2,3 & 62,5 & 33,3 & 4,2 \\
$\begin{array}{l}\text { por descubrimiento, la modelización, el enfoque } \\
\text { ciencia tecnología y sociedad (CTS), entre otras. }\end{array}$ & & & & & & \\
$\begin{array}{l}\text { Situaciones de aprendizajes que contribuyan a que el } \\
\text { estudiantado desarrolle el pensamiento crítico y sistémico. }\end{array}$ & 62,5 & 34,1 & 3,4 & 73,6 & 25,0 & 1,4 \\
$\begin{array}{l}\text { Situaciones de aprendizajes que contribuyan a que el } \\
\text { estudiantado se apropie de las tecnologías digitales. }\end{array}$ & 55,7 & 35,2 & 9,1 & 72,2 & 16,7 & 11,1 \\
$\begin{array}{l}\text { Situaciones de aprendizajes que contribuyan a que el } \\
\text { estudiantado desarrolle estilos de vida saludables. }\end{array}$ & 72,7 & 21,6 & 5,7 & 69,4 & 25,0 & 5,6 \\
\hline
\end{tabular}

Nota: Cuestionario de docentes de ciencias naturales y matemática. Elaboración propia.

Por otra parte, sobre la dimensión búsqueda de información e investigación la opinión de los y las docentes acerca del interés por capacitarse sigue siendo alto, superior a un $90 \%$, tanto para matemática, como para ciencias (Tabla 3).

Tabla 3: Grado de interés del cuerpo docente de química, física, biología y matemática en recibir capacitaciones en búsqueda de información e investigación, Dirección Regional de Educación de Heredia, 2018

\begin{tabular}{llcccccc}
\hline & \multicolumn{5}{c}{ Grado de interés } \\
\cline { 2 - 7 } & \multicolumn{3}{c}{ Ciencias } & \multicolumn{3}{c}{ Matemática } \\
\cline { 2 - 7 } Criterios & $\begin{array}{c}\text { Alto } \\
\%\end{array}$ & $\begin{array}{c}\text { Medio } \\
\%\end{array}$ & $\begin{array}{c}\text { Bajo } \\
\%\end{array}$ & $\begin{array}{c}\text { Alto } \\
\%\end{array}$ & $\begin{array}{c}\text { Medio } \\
\%\end{array}$ & $\begin{array}{c}\text { Bajo } \\
\%\end{array}$ \\
\hline $\begin{array}{l}\text { Desarrollo de estrategias para la apropiación de las } \\
\text { tecnologías digitales. }\end{array}$ & 52,3 & 39,8 & 8,0 & 73,6 & 19,4 & 6,9 \\
$\begin{array}{l}\text { Desarrollo habilidades en el estudiantado para la búsqueda } \\
\text { de la información, para la investigación y para promover } \\
\text { habilidades metacognitivas que le permitan resolver } \\
\text { situaciones cotidianas con razonamiento científico. }\end{array}$ & 63,6 & 31,8 & 4,5 & 59,7 & 36,1 & 4,2 \\
\hline
\end{tabular}

Nota: Cuestionario de docentes de ciencias y matemática. Elaboración propia. 
http://doi.org/10.15359/ree.24-3.23

http://www.una.ac.cr/educare

educare@una.ac.cr

La dimensión de procedimientos de evaluación alcanzó el mayor porcentaje de interés de capacitación, con porcentajes superiores al $96 \%$ para los aspectos relacionados con las rúbricas para evaluar el trabajo cotidiano y construcción de exámenes, así como la coherencia entre los criterios de evaluación, las situaciones de aprendizaje y los indicadores (Tabla 4).

Tabla 4: Grado de interés de docentes de física, química, biología y matemática en recibir capacitaciones en procedimientos de evaluación, Dirección Regional de Educación de Heredia, 2018

\begin{tabular}{lcccccc}
\hline & \multicolumn{5}{c}{ Grado de interés } \\
\cline { 2 - 7 } & \multicolumn{4}{c}{ Ciencias } \\
\cline { 2 - 7 } Criterios & $\begin{array}{c}\text { Alto } \\
\%\end{array}$ & $\begin{array}{c}\text { Medio } \\
\%\end{array}$ & $\begin{array}{c}\text { Bajo } \\
\%\end{array}$ & $\begin{array}{c}\text { Alto } \\
\%\end{array}$ & $\begin{array}{c}\text { Medio } \\
\%\end{array}$ & $\begin{array}{c}\text { Bajo } \\
\%\end{array}$ \\
\hline $\begin{array}{l}\text { Evaluación del proceso según su función } \\
\text { (diagnóstica, formativa y sumativa) aplicando la } \\
\text { autoevaluación y coevaluación. }\end{array}$ & 59,1 & 29,5 & 11,4 & 55,6 & 38,9 & 5,6 \\
$\begin{array}{l}\text { Rúbricas para evaluar el trabajo cotidiano y } \\
\text { construcción de ítems para los exámenes. }\end{array}$ & 73,9 & 23,9 & 2,3 & 80,6 & 18,1 & 1,4 \\
$\begin{array}{l}\text { Coherencia entre los criterios de evaluación, las } \\
\text { situaciones de aprendizaje y los indicadores. }\end{array}$ & 75,0 & 21,6 & 3,4 & 80,6 & 18,1 & 1,4 \\
\hline
\end{tabular}

Nota: Cuestionario de docentes de ciencias naturales y matemática. Elaboración propia.

\section{Uso de las tecnologías}

Con respecto a las habilidades de docentes para el manejo de las TIC en su práctica profesional, según los datos de la Figura 2 y la Figura 3 de ciencias y matemática respectivamente, el $85 \%$ de docentes de ciencias y el $82 \%$ de matemática se ubican como hábiles y muy hábiles en buscar información en diferentes medios electrónicos, así como en comunicarse por este medio y usar fuentes de información y de recursos para el aprendizaje.

Sobre las habilidades cognitivas para utilizar softwares educativos, un $62 \%$ de docentes de ciencias naturales se considera hábil y muy hábil, así como el 65\% de docentes de matemática, en términos generales, se puede afirmar que tiene un buen nivel de estas habilidades. Sin embargo, llama la atención que en lo que respecta a la utilización de simuladores, existe un $55 \%$ de docentes de ciencias y $68 \%$ de matemática se consideran poco hábiles o no los usan. 
http://doi.org/10.15359/ree.24-3.23

http://www.una.ac.cr/educare educare@una.ac.cr

Figura 2: Nivel de habilidad identificado por los docentes de ciencias naturales de secundaria, para el manejo de algunos recursos tecnológicos. Dirección Regional de Educación de Heredia, 2018

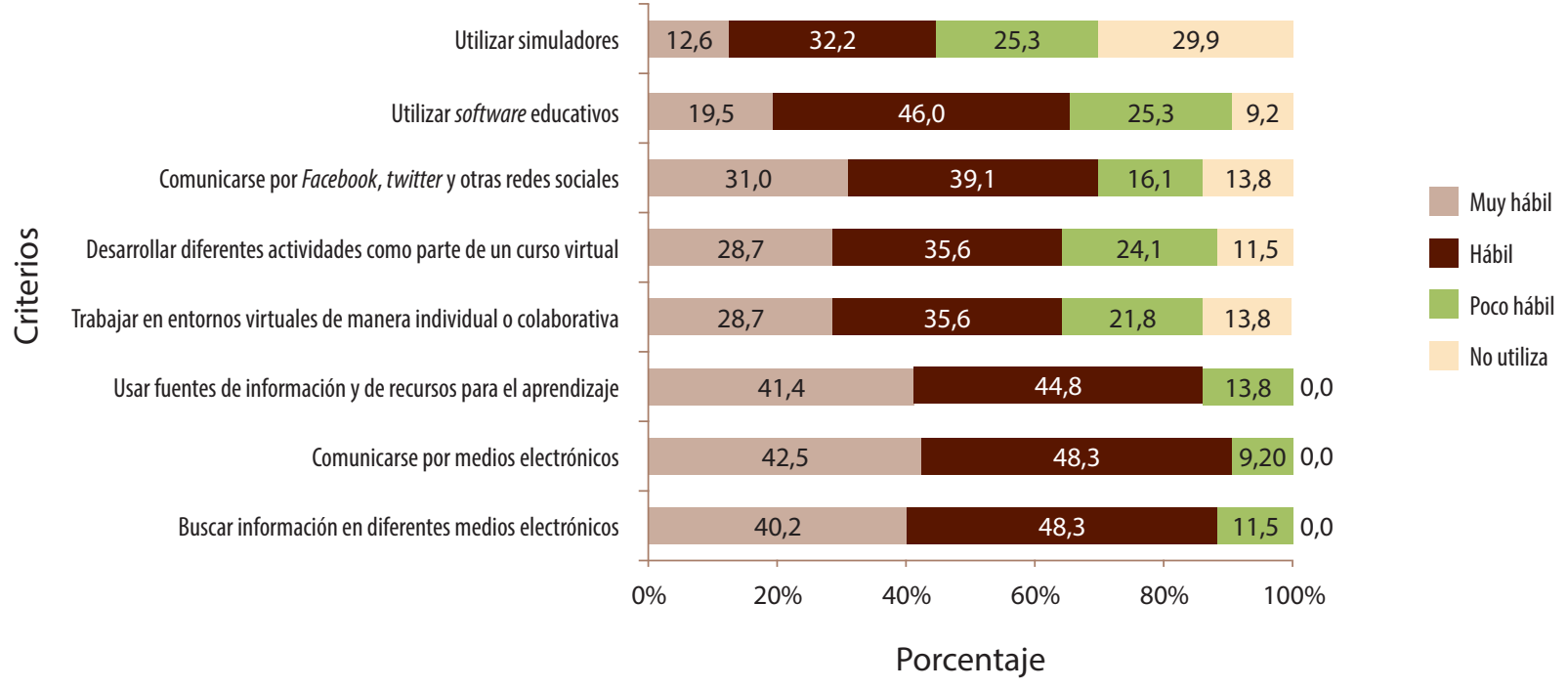

Nota: Elaboración propia.

Figura 3: Nivel de habilidad identificado por los docentes de matemática de secundaria, para el manejo de algunos recursos tecnológicos. Dirección Regional de Educación de Heredia, 2018

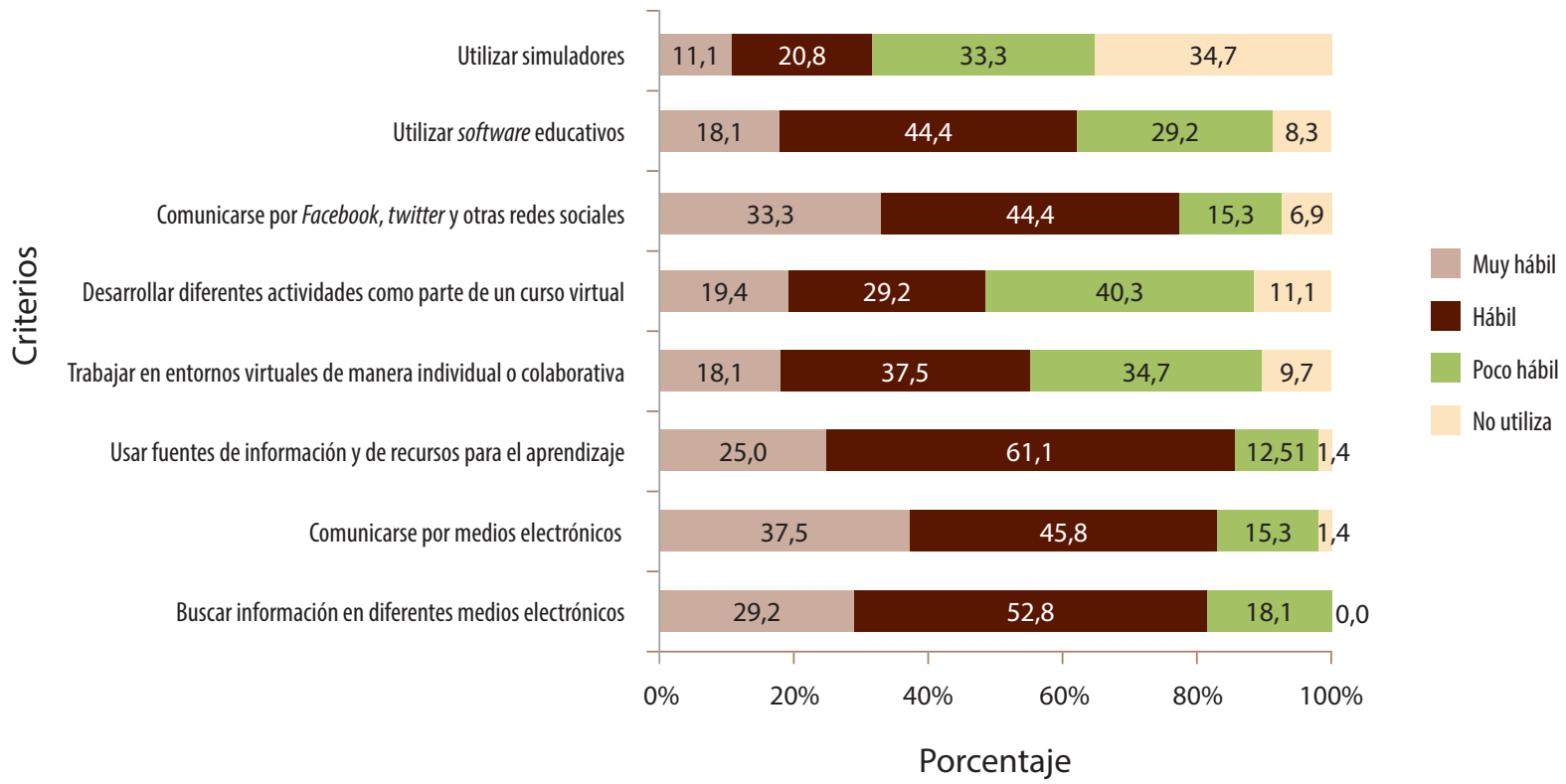

Nota: Elaboración propia. 
http://doi.org/10.15359/ree.24-3.23

http://www.una.ac.cr/educare

educare@una.ac.cr

Con respecto a la utilización que hace el personal docente de los recursos y herramientas electrónicas para desarrollar sus clases (Tabla 5), se puede afirmar que en el caso de matemática la utilización de equipo de cómputo (computadora portátil), proyectores multimedia (73,6\%) y calculadora (95\%), son los más utilizados con porcentajes superiores al $70 \%$. En el caso de ciencias naturales, los más utilizados son buscadores de internet, google académico u otros sitios confiables para buscar información (78,2\%), equipo de cómputo (computadora portátil) y proyectores multimedia $(75,9 \%)$, teléfono celular $(78,2 \%)$ y calculadora $(80.5 \%)$. En términos generales, se nota que hay mayor tendencia a utilizar recursos y herramientas por parte de docentes de ciencias en relación con los de matemática.

Tabla 5: Recursos y herramientas electrónicas utilizadas por el cuerpo docente de matemática y ciencias naturales de secundaria para desarrollar sus clases. Dirección Regional de Heredia, 2018

\begin{tabular}{|c|c|c|c|c|}
\hline \multirow[t]{2}{*}{ Recursos o herramienta tecnológica } & \multicolumn{2}{|c|}{ Matemática } & \multicolumn{2}{|l|}{ Ciencias } \\
\hline & Frecuencia Absoluta & Porcentaje & Frecuencia Absoluta & Porcentaje \\
\hline Messenger o Chats & 10 & 13,9 & 22 & 22,3 \\
\hline Redes Sociales & 5 & 6,9 & 11 & 12,6 \\
\hline $\begin{array}{l}\text { Buscadores de Internet, Google } \\
\text { académico u otros sitios confiables para } \\
\text { buscar información }\end{array}$ & 39 & 54,2 & 68 & 78,2 \\
\hline Aplicaciones interactivas (Apps) & 19 & 26,4 & 35 & 40,2 \\
\hline Bases de datos científicas & 3 & 4,2 & 18 & 20,7 \\
\hline $\begin{array}{l}\text { Equipo de cómputo (computadora } \\
\text { portátil) y proyectores multimedia }\end{array}$ & 53 & 73,6 & 66 & 75,9 \\
\hline Equipo de audio & 17 & 23,6 & 45 & 51,7 \\
\hline $\begin{array}{l}\text { Herramientas básicas de Microsoft Office } \\
\text { (Word, Excel, Power Point, Publisher) }\end{array}$ & 39 & 54,2 & 59 & 67,8 \\
\hline Descarga de recursos audiovisuales & 23 & 31,9 & 48 & 55,2 \\
\hline Plataformas virtuales & 8 & 11,1 & 18 & 20,7 \\
\hline Software educativos & 26 & 36,1 & 16 & 18,4 \\
\hline Simuladores & 2 & 2,8 & 14 & 16,1 \\
\hline Teléfono celular & 37 & 51,4 & 68 & 78,2 \\
\hline Calculadora & 69 & 95,8 & 70 & 80,5 \\
\hline
\end{tabular}

Nota: Cuestionario de docentes de ciencias y matemática. Elaboración propia.

20 Adriana Zúniga-Meléndez, Alejandro Durán-Apuy, Jesennia Chavarría-Vásquez, Ronny Gamboa-Araya, Ana Francis Carballo-Arce, Xinia Vargas-González, Nelson Campos-Quesada, Cecilia Sevilla-Solano e Isabel Torres-Salas 
http://doi.org/10.15359/ree.24-3.23

\section{Retos y desafíos de la práctica docente}

Como parte del encuentro realizado con los grupos docentes, manifestaron, de forma oral, algunas de las dificultades que a la fecha han enfrentado en la implementación de los nuevos programas de estudio de la política Educar para una nueva ciudadanía. La principal inquietud se centra en el porcentaje asignado al rubro de trabajo cotidiano, de acuerdo con su opinión, los espacios de aula no son los adecuados para el desarrollo de una propuesta didáctica diferente, tal como lo requiere el nuevo abordaje educativo.

Según indican, la gran cantidad de actividades extracurriculares que deben desarrollar les impide avocarse en el diseño de estrategias de aula acordes con el nuevo modelo. Consideran además, que hay una incertidumbre en cuanto a la calidad y conocimientos adquiridos por el estudiantado y el sistema de evaluación que se está empleando. Ello los lleva a cuestionar cuál será el desempeño de este estudiantado en pruebas estandarizadas. El cuerpo docente indicó, además, la falencia de estrategias de evaluación que permitan valorar el trabajo cotidiano, manifestó la preocupación al tiempo y atención requerida para la atención de estudiantes con adecuaciones significativas, sobre todo en grupos muy numerosos.

\section{Discusión de resultados}

Con base en la caracterización general realizada al personal docente, se puede evidenciar que son profesionales con amplia experiencia en su quehacer y que además ostentan altos grados académicos, reconocidos por el principal ente empleador (Ministerio de Educación Pública). Esta situación se constituye en una fortaleza, pues una mayor preparación académica acompañada de una amplia experiencia, potencian mejores prácticas de clase, promoviendo resultados de aprendizaje más significativos. No obstante, podría convertirse en una debilidad, si el personal docente maneja esquemas o estructuras rígidas, que no le permitan asimilar el cambio propuesto desde la nueva política curricular.

\section{Saber disciplinar}

Estos resultados coinciden con lo esperado, pues en matemática, si se analizan los anteriores programas de estudio en el confronte con vigentes, la estadística y la probabilidad pasaron de ser temáticas aisladas en secundaria, para convertirse en un área disciplinar de la actual propuesta curricular, la cual es desarrollada desde primaria y, por lo tanto, su profundidad y alcances para la educación secundaria son significativamente mayores. En el caso de la biología, química y física, además de ser temáticas muy generales y teóricas, tienen un alto grado de dificultad al momento de generar demostraciones que permitan una mayor comprensión. 
http://doi.org/10.15359/ree.24-3.23

http://www.una.ac.cr/educare

educare@una.ac.cr

Nuevamente, al comparar estos temas con las exigencias del currículo educativo actual, se puede evidenciar total coherencia, pues el programa de estudio ha establecido no solo como contenido sino como enfoque metodológico la resolución de problemas, y la contextualización activa; de manera que queda justificado el que aparezcan temas de desarrollo teórico y abstracto.

\section{Saber pedagógico}

En relación con el análisis sobre los saberes pedagógicos, se logró constatar que para ambos grupos docentes (ciencias naturales y matemática), todos los criterios analizados presentaron porcentajes medios y altos de interés. Esto refleja claramente una intencionalidad por parte del cuerpo docente a involucrarse en procesos de formación y actualización permanente, en aspectos relacionados con la planificación, mediación y evaluación, así como en temas vinculados al uso de las tecnologías de la información y la comunicación, todo esto, con la finalidad de fortalecer su perfil profesional y desarrollar mejores prácticas de aula.

En lo que respecta a la planificación didáctica, la necesidad de implementar metodologías activas, que promuevan habilidades para la argumentación, el debate y la criticidad, son elementos indispensables para el abordaje de las clases desde una metodología indagatoria, la cual es el sustento metodológico en el nuevo diseño programático adoptado recientemente por el MEP. Esta metodología supone que el estudiantado, desde un papel más protagónico, desarrolle niveles cognitivos que trasciendan los contenidos conceptuales hacia los contenidos procedimentales y actitudinales, y se aproxime, así, al desarrollo de habilidades propias de la metodología y del pensamiento científico.

De igual manera, en los nuevos planes de estudio impulsados por el MEP, se incluye, como uno de los ejes curriculares medulares, las distintas formas de pensamiento, dentro de las cuales se enmarcan el pensamiento crítico y sistémico. Lograr el desarrollo exitoso de habilidades en estas líneas de pensamiento, implica para los grupos docentes no solo poseer un conocimiento muy sólido a nivel disciplinar, sino además desarrollar una capacidad creativa a la hora de diseñar y ejecutar la propuesta de clase y las respectivas estrategias de evaluación.

El fin de este enfoque es que el estudiantado pueda enfrentarse a situaciones contextualizadas, en las que se requiera del análisis, la inferencia, la argumentación, la evaluación, la interpretación, así como elementos de comprensión y toma de decisión. Si bien es cierto, se presume que todas estas habilidades deberían estar apropiadas en cada docente, se interpreta, a partir de sus necesidades manifiestas, que, probablemente, en el proceso de formación inicial del profesorado, este tipo de habilidades no fueron completamente abordadas.

Por otra parte, las situaciones de aprendizaje deben incorporar una serie de elementos y herramientas para lograr un objetivo concreto de aprendizaje. En el contexto educativo actual, una de las principales herramientas a considerarse es la apropiación de las tecnologías digitales, 
http://doi.org/10.15359/ree.24-3.23

las cuales pretenden la integración del estudiantado a la sociedad del conocimiento como parte de la ciudadanía planetaria. Esta situación marca un momento en el que el cuerpo docente debe redefinir sus prácticas de aula, para poder responder a esta nueva forma de abordar los procesos de enseñanza y aprendizaje en general, y de la disciplina en particular. De ahí que esta visión sea congruente con lo manifestado por los cuerpos docentes, en cuanto a un alto grado de interés en fortalecer sus habilidades para incorporar las tecnologías en sus actividades de mediación.

En la formación de una ciudadanía planetaria, debe promoverse una concepción integral de la persona desde nuevas formas de convivir en el mundo, que incorporen, a su vez, estilos de vida saludables. Es decir, se concibe una persona con capacidad de reconocer los riesgos socioambientales que afectan todas las formas de vida. Una de las metas de la nueva política curricular es, precisamente, lograr el desarrollo holístico de la persona; contempla, para esto, que desde el proceso educativo se promueva la apropiación de experiencias que le permitan enfrentar problemas, considerando las relaciones entre los factores socioculturales, económicos y ambientales de su entorno. Se promueven. además, habilidades para el cuidado de la salud, tanto de forma personal como comunitaria.

Por otra parte, en un mundo globalizado, existen un sinnúmero de fuentes de información que pueden ser consultadas con relativa facilidad. No obstante, aun cuando esto podría considerarse una situación muy favorable, se requiere poseer un juicio discriminante que permita diferenciar aquella información con validez científica de la que no la tiene. Esto implica, en primera instancia, la capacidad de reconocer espacios de búsqueda confiables, que cumplan con los criterios de rigurosidad científica. Por otra parte, es necesario tener destrezas en identificar, seleccionar y procesar la información consultada, atendiendo en todo momento los objetivos propuestos a la hora de plantear la búsqueda.

Las posibilidades de incorporar, dentro de las situaciones de aprendizaje, dispositivos electrónicos como computadoras, celulares, tabletas; así como recursos digitales, tales como simuladores, entornos virtuales, software educativo, aplicaciones, etc., motivan al personal docente a estar en un continuo proceso de actualización, a fin de desarrollar en el estudiantado el interés en apropiar este tipo de herramientas para potenciar las habilidades en la búsqueda y uso de información científicamente válida.

En cuanto al proceso evaluativo, tradicionalmente ha existido una tendencia hacia una evaluación sumativa, orientada hacia la verificación de resultados de aprendizajes puntuales, y han dejado de lado, en muchos casos, el proceso mediante el cual se obtuvieron dichos aprendizajes. En el contexto educativo actual, los procesos de evaluación deben atenderse desde una función más dialógica, diagnóstica y metacognitiva, garantizándose con esto que haya un acompañamiento más cercano del cuerpo docente a las etapas del proceso de aprendizaje, en el que está inmerso el estudiantado. Para alcanzar estas metas se requiere que el 
http://doi.org/10.15359/ree.24-3.23

http://www.una.ac.cr/educare

educare@una.ac.cr

personal docente tenga una preparación para atender la diversidad de estilos de aprendizaje de las personas aprendientes. Esta postura concuerda con el alto nivel de interés del profesorado en recibir capacitación en temáticas relacionadas con la evaluación de aprendizajes.

Dentro de los aspectos de evaluación que podrían representar un reto significativo en la labor docente, se encuentra el trabajo cotidiano. Esto, considerando que para lograr evaluar el desempeño estudiantil en este proceso áulico se requieren una serie de parámetros e indicadores de valoración, que en su construcción son complejos, dado que intentan evidenciar cada uno de los diferentes niveles cognitivos, adquiridos durante la secuencia de aprendizaje.

La situación anterior se hace más palpable, a partir del cambio, en la distribución de los rubros de evaluación propuestos por el MEP en el año 2018 (Artículos 36 y 37 del Reglamento de evaluación de los aprendizajes), donde se le asigna al trabajo cotidiano un valor porcentual muy alto. Por ejemplo, en el III Ciclo de Educación General Básica, el valor del trabajo cotidiano, pasó de un $25 \%$ a un 45\%, mientras que en el Ciclo Diversificado pasó de un 10\% a un 35\% de la calificación final. Por lo tanto, este cambio representa un reto importante, en cuanto a la elaboración de actividades de mediación en el aula y en la construcción de rúbricas que permitan ser objetivas en la evaluación del trabajo en clase.

Otro aspecto que considerar en los procesos evaluativos es la coherencia que debe existir entre los objetivos propuestos, las situaciones de aprendizaje y los indicadores de evaluación. Los objetivos nos plantean una ruta en la que se establecen, con claridad, los resultados de aprendizaje esperados; las situaciones de aprendizaje se transforman en el medio por el cual se alcanzarán estos resultados y los indicadores de evaluación se convierten en el mecanismo para la comprobación y retroalimentación del proceso de aprendizaje, considerando además características y necesidades individuales estudiantiles. En el nuevo enfoque de evaluación por habilidades, lograr la coherencia entre estos tres aspectos supone un desafío mayor en términos de planificación y evaluación, porque las habilidades integran elementos dentro del saber saber, saber hacer y saber ser. No es extraño que el profesorado manifieste un alto grado de interés en fortalecer sus habilidades para implementar procesos articulados entre la planificación y la evaluación.

\section{Uso de las tecnologías}

La incorporación de las tecnologías en los espacios áulicos exige que el profesorado desempeñe nuevas funciones pedagogías para las que diseñe ambientes de aprendizaje menos tradicionales, más dinámicos, en los que se promueva el aprendizaje cooperativo, el trabajo colaborativo y se potencie el desarrollo de habilidades para el manejo de las tecnologías. Para esto, el cuerpo docente debe seleccionar y utilizar, de forma apropiada, una serie de equipos y recursos tecnológicos con el objetivo de promover el aprendizaje. 
http://doi.org/10.15359/ree.24-3.23

El uso de los recursos tecnológicos tradicionales como calculadora, equipo de cómputo, proyectores de multimedios, y paquete de Microsoft Office pueden transformarse en herramientas útiles para el desarrollo de una clase, al permitir resolver problemas con mayor rapidez, presentar información de manera más atractiva, consultar información en el momento cuando surgen las inquietudes. No obstante, el personal docente debe anhelar un conocimiento variado sobre software y herramientas tecnologías que sean de fácil acceso y bajo costo, diseñadas con fines educativos o que puedan ser adaptadas para este fin. En este caso, recursos como simuladores, softwares educativos, bases de datos científicas y aplicaciones interactivas promueven una participación más activa del estudiantado y permiten el desarrollo de experiencias colaborativas de indagación, con las cuales se promueven habilidades científicas y tecnológicas, por lo que se esperaría que el cuerpo docente las utilizara al menos con la misma frecuencia de uso de los recursos tradicionales.

De igual forma, los softwares educativos y los simuladores se transforman en entornos virtuales interactivos que permiten recrear fenómenos o procesos, facilitando la exploración, la observación y manipulación, así como la toma de decisiones en situaciones que difícilmente son accesibles en la realidad. Muchos de estos softwares y simuladores pueden ser incluso utilizados a través de los teléfonos inteligentes que hoy en día se encuentran al alcance de la mayoría de estudiantes.

No obstante, de acuerdo con los manifestado por los grupos docentes, el uso de estos recursos tecnológicos (softwares educativos y los simuladores) en su práctica es limitado, lo cual atribuyen al bajo nivel de habilidad en el uso de estas herramientas en particular. Además, según su criterio, la incorporación de este tipo de recursos requiere una mayor inversión de tiempo en la planificación, diseño y ejecución de estrategias de mediación.

Esta situación señala la necesidad de potenciar este tipo de habilidades en su formación inicial y fortalecerlas a través de procesos de actualización profesional.

\section{Retos manifestados por los grupos docentes}

Con base en el análisis de los retos manifestados por docentes de secundaria, durante el encuentro realizado, el grupo de trabajo puede observar una preocupación por la implementación de la nueva propuesta curricular, tanto en el enfoque metodológico como en los mecanismos de evaluación, y no tanto en áreas disciplinarias en las cuales se sienten capaces.

Estas preocupaciones son coherentes con la ruptura de paradigma que propone el actual currículo educativo, que implica la transición de una educación centrada en quien enseña contenidos, hacia una educación centrada en el estudiantado y las habilidades, donde el conocimiento no es un fin, sino una herramienta para el desarrollo de habilidades científicas. 
http://doi.org/10.15359/ree.24-3.23

http://www.una.ac.cr/educare

educare@una.ac.cr

Algunas de las estrategias pedagógicas que podrían ser utilizadas para el abordaje de la nueva propuesta curricular son: el aprendizaje activo, la evaluación auténtica, el aprendizaje por habilidades.

\section{Conclusiones}

La nueva transformación curricular denominada "Educar para una nueva ciudadanía" propuesta por el Ministerio de Educación Pública de Costa Rica, establece la educación basada en los derechos humanos, sustentada en tres pilares, a saber: la educación para el desarrollo sostenible, la ciudadanía digital con equidad social y el fortalecimiento de una ciudadanía planetaria con identidad nacional (MEP, 2015. P. 10). Esta propuesta se basa en el desarrollo de habilidades que le permiten al estudiantado resolver situaciones reales, mediante la aplicación de los conocimientos adquiridos, los cuales sirven como base para la generación de autoconocimiento. Lo anterior se logra mediante la implementación de estrategias de aula orientadas a promover la reflexión, el modelado y la sistematización del conocimiento, al enfrentar situaciones significativas ligadas al entorno.

Como consecuencia del análisis de esta reforma curricular, se hacen evidentes los retos que implica su implementación, ya que la persona docente debe transformar su acción educativa, replanteando la manera en que realiza la mediación pedagógica, de forma tal que esta promueva el desarrollo de habilidades en el estudiantado, teniendo claro el rol docente en este nuevo paradigma.

El diagnóstico realizado a docentes de ciencias naturales y matemática evidencia las siguientes necesidades de capacitación:

\section{- Saber disciplinar:}

En relación con los temas de matemática se concluye que la capacitación de matemática debe enfocarse con mayor fuerza en estadística y probabilidad, también debe incluir geometría y funciones matemáticas.

- En lo referente a biología, la capacitación debe centrarse en los siguientes temas: prioritariamente ecología, seguido de evolución, genética, célula, ciclo celular y cuidado del ambiente y, biotecnología.

- Para el área de física, el mayor interés de capacitación corresponde al tema de cinemática, seguido por física moderna y, por último, trabajo, energía y electricidad.

- En el caso de química, se demanda capacitación, prioritariamente, en tópicos como compuestos químicos, enlaces químicos, seguidos por estequiometría y química orgánica (nomenclatura y grupos funcionales). 
http://doi.org/10.15359/ree.24-3.23

\section{- Saber pedagógico}

En esta área de conocimiento el profesorado manifestó necesitar capacitación concentrada en el ámbito de evaluación, dado que manifiesta una gran preocupación por el alto porcentaje destinado al trabajo cotidiano y no cuentan con las estrategias de evaluación adecuadas para calificar este rubro. Además, manifestó interés en ser capacitado en lo referente a planificación didáctica y mediación pedagógica.

\section{Uso de la tecnología:}

La incorporación de los recursos tecnológicos en el contexto aúlico está vinculada con el grado de habilidad que poseen los grupos docentes en el manejo de estos recursos.

El diagnóstico demuestra que el personal docente se autodenomina como hábil y muy hábil en el manejo de las tecnologías de la información y la comunicación (TICS).

Los simuladores y programas informáticos son los recursos menos utilizados por docentes, debido a que, según lo manifestado, poseen poco conocimiento en el uso de estos.

La planificación de estrategias de mediación basadas en recursos tecnológicos requieren de una inversión de tiempo, materiales y destrezas mayores a otro tipo de recursos más tradicionales, según lo expresado por los grupos docentes.

\section{Retos manifestados por el personal docente}

El contraste entre los intereses de capacitación manifestados en el instrumento de diagnóstico y los retos manifestados de forma verbal por el personal docente permite concluir que la capacitación en áreas disciplinarias debe estar enfocada al diseño de estrategias de mediación, interdisciplinarias y contextualizadas, con un enfoque de aprendizaje activo, así como en el diseño de procesos evaluativos que sirvan como instrumentos de diagnóstico para determinar, en el estudiantado, el grado de apropiación de las habilidades de pensamiento científico y matemático, y el logro de los resultados de aprendizaje establecidos para cada tema

De forma general, los resultados obtenidos del diagnóstico evidencian, una vez más, las necesidades de una capacitación permanente a un cuerpo docente perteneciente a áreas del conocimiento que evolucionan de forma muy dinámica en el contexto actual y que deben responder a la formación oportuna de una ciudadanía planetaria como la concebida en la reforma curricular planteada por el MEP-Costa Rica.

El esfuerzo realizado por el Ministerio de Educación Pública, al plantear una reforma curricular que potencia las habilidades de pensamiento científico, solo se puede concretar a través de procesos de capacitación, que permitan al personal docente la compresión de la 
http://doi.org/10.15359/ree.24-3.23

http://www.una.ac.cr/educare

educare@una.ac.cr

trascendencia del cambio que se persigue, y el papel determinante que las áreas de ciencias naturales y matemáticas juegan en el desarrollo de un país. Se pone de manifiesto, además, el papel que debe cumplir la universidad como protagonista en el acompañamiento de profesionales a través de procesos de formación y actualización continua en las distintas áreas del conocimiento.

\section{Referencias}

Alfaro Varela, G. y Villegas, L. R. (2010). La educación científica en Costa Rica. Tercer Informe Estado de la Educación. Programa Estado de la Nación.

Aristizabal-Almanza, J. L., Ramos-Monobe, A., Chirino-Barceló, V. (2018). Aprendizaje activo para el desarrollo de la psicomotricidad y el trabajo en equipo. Revista Electrónica Educare, 22(1),1-26. http://dx.doi.org/10.15359/ree.22-1.16

Bosch, H. E., Di Blasi, M. A., Pelem, M. E., Bergero, M. S., Carvajal, L. y Geromini, N. S. (2011). Nuevo paradigma pedagógico para enseñanza de ciencias y matemática. Avances ciencias e ingeniería, 2(3), 131-140. https://dialnet.unirioja.es/servlet/articulo?codigo=3752199

Bozu, Z. e Imbernón, F. (2016). El presente y el futuro de la formación permanente docente del profesoradouniversitario.Educaciónyciencia,5(45),94-105.http://www.educacionyciencia. org/index.php/educacionyciencia/article/view/387

Klimenko, O. (2008). La creatividad como un desafío para la educación del siglo XXI. Educación y Educadores, 11(2), 191-210. http://www.scielo.org.co/pdf/eded/v11n2/v11n2a12.pdf

Macedo, B. (2016). Educación científica. UNESCO. http://repositorio.minedu.gob.pe/ handle/123456789/5025

Ministerio de Educación Pública. (2012). Programas de Estudio de Matemáticas. Autor. https:// www.mep.go.cr/sites/default/files/programadeestudio/programas/matematica.pdf

Ministerio de Educación Pública. (2015). Fundamentación pedagógica de la transformación curricular. Bajo la versión de educar para una nueva ciudadanía. Autor. http://www.mep. go.cr/educar-para-nueva-ciudadania

Ministerio de Educación Pública.(2017a). Programas de estudio de química educación diversificada. Autor. $\quad$ http://www.mep.go.cr/sites/default/files/programadeestudio/programas/ quimica2018.pdf

Ministerio de Educación Pública. (2017b). Política educativa "La persona: Centro del proceso educativo y sujeto transformador de la sociedad". Autor. http://www.mep.go.cr/sites/ default/files/page/adjuntos/politicaeducativa.pdf 
http://doi.org/10.15359/ree.24-3.23 http://www.una.ac.cr/educare educare@una.ac.cr

Ministerio de Educación Pública. (2017c). Educar para la nueva ciudadanía. Programa de estudio de Ciencias de tercer ciclo de educación general básica. Autor. https://www.mep.go.cr/sites/ default/files/programadeestudio/programas/ciencias3ciclo.pdf

Palomares Ruiz, A. (2004). Profesorado y educación para la diversidad en el siglo XXI. Ediciones de la Universidad de Castilla-La Mancha.

Peñaherrera León, M., Ortiz Colón, A. y Cobos Alvarado, F. (2013). ¿Cómo promover la educación científica en el alumnado de primaria? Una experiencia desde el contexto ecuatoriano. Revista Eureka sobre Enseñanza y Divulgación de las Ciencias, 10(2), 199-209. https://doi. org/10.25267/Rev Eureka ensen divulg cienc.2013.v10.i2.07

Programa Estado de la Nación. (2011). Estado de la Educación Costarricense 3. Autor. http:// repositorio.conare.ac.cr/bitstream/handle/20.500.12337/675/Estado\%20de\%20la\%20 Educaci\%c3\%b3n\%203.pdf?sequence=22\&isAllowed $=y$

Programa Estado de la Nación. (2014). Estado de la ciencia, la tecnología y la innovación. Autor. http://eccti.or.cr/media/documentos/ECTI-Book.pdf

Programa Estado de la Nación. (2015). Estado de la educación costarricens 5. Autor. http://repositorio.conare.ac.cr/bitstream/handle/20.500.12337/669/ESTADOEDUCACION-5-2015.pdf?sequence=18\&isAllowed=y

Programa Estado de la Nación. (2017). Sexto informe estado de la educación. Autor. http:// repositorio.conare.ac.cr/handle/20.500.12337/665

Torres Salas, M. I. (2010). La enseñanza tradicional de las ciencias versus las nuevas tendencias educativas. Revista Electrónica Educare, 14(1), 131-142. https://doi.org/10.15359/ree.14-1.11

UNESCO. (2017). Informe de seguimiento de la educación en el mundo 2016: La educación al servicio de los pueblos y el planeta: Creación de futuros sostenibles para todos. Ediciones UNESCO. https://unesdoc.unesco.org/ark:/48223/pf0000248526

Valverde, G. y Näslund-Hadley, E. (2010). La condición de la educación en matemáticas y ciencias naturales en América Latina y el Caribe. BID. https://publications.iadb.org/ handle/11319/2757

Vélez Chablé, G. (2006). El docente ante los retos educativos del siglo XXI. Pampedia, 2, 55-59. https:// www.academia.edu/20417364/6-El-docente-ante-los-retos-educativos-del-siglo-XXI

Zamora Araya, J. A. (2015). Análisis de confiabilidad de la Prueba de Diagnóstico Matemática en la Universidad Nacional de Costa Rica. Actualidades en Psicología, 29(119), 153-165. https:// doi.org/10.15517/ap.v29i119.18693 